\title{
Tacrolimus or clobetasol for treatment of oral lichen planus
}

\author{
Abstracted from \\ Chamani G, Rad M, Zarei MR, Lotfi S, Sadeghi M, Ahmadi Z. \\ Efficacy of tacrolimus and clobetasol in the treatment of oral lichen planus: \\ a systematic review and meta-analysis. Int / Dermatol 2015; 54: 996-1004. \\ doi: 10.1111/ijd.12925. Epub 2015 Jul 23. PubMed PMID: 26204904. \\ Address for correspondence: Maryam Rad, Oral and Dental Diseases Research Center, \\ Kerman University of Medical Sciences, Kosar Boulevard, Kerman 7618836555 Iran. E-mail: rad_1152@yahoo.com
}

\section{Question: Is topical tacrolimus or clobetasol effective in treatment of oral lichen planus (OLP)?}

Data sources Pubmed, the Cochrane library, Scopus, Science Direct and two publishing company journals between 1998 and 2012. Study selection Randomised controlled trials (RCTs) where the population included patients having OLP and the interventions were the use of clobetasol or tacrolimus compared to another intervention, while the outcome was improvement in clinical status.

Data extraction and synthesis Two authors working independently assessed for inclusion and performed data extraction. Quality was evaluated using Critical Appraisal Skills Programme (CASP) worksheets (http://www.casp-uk.net/). The treatment effect was calculated using $\mathrm{OR}$ and then pooled using a fixed model since heterogeneity was calculated as very low.

Results Ten studies were included; five studies involved clobetasol and five involved tacrolimus. Two meta-analyses were presented. The odds ratio for improvement for clobetasol was 1.21 (95\% Cl; 0.48 $3.05)$ and $8.09(95 \% \mathrm{Cl} ; 3.77$ - 17.38) for tacrolimus.

Conclusions The authors concluded that using clobetasol or tacrolimus increases the odds of improvement of OLP lesions and therefore they are effective treatment for the condition, and go on to recommend tacrolimus as first-line therapy.

\section{Commentary}

Initially in this systematic review the authors wanted to compare the efficacy of topical tacrolimus to clobetasol for the treatment of oral lichen planus (OLP) claiming that such a review does not exist.

Four databases were searched until 12/2012 with no language restriction. There is some indication of hand searching and no mention of gray literature. They included RCTs where the intervention was the use of tacrolimus or clobetasol compared to another intervention, and the outcome was patient improvement.

Two authors assessed articles for inclusion. Even though the authors claimed they followed the 'Cochrane Guidance for Systematic Reviews', they assessed the quality of the included studies using CASP and proceeded to include only ones with a score of $\geq 6$. No comparison is possible between that particular scoring system and the more widely used Cochrane Collaboration's tool for assessing risk of bias.

Of the ten studies, three compared either 0.025 or $0.05 \%$ topical clobetasol to another steroid $(n=120)$; one compared clobetasol with mesalazine 5\% $(n=25)$; and one clobetasol to cyclosporine $(n=39)$. Of the remaining five studies, four compared topical tacrolimus $0.1 \%$ to clobetasol $0.05 \%(n=131)$, and one compared tacrolimus to triamcinolone $0.1 \%(n=60)$. Patient treatment varied between four to 16 weeks.

The authors calculated OR and performed two meta-analyses using the primary outcome: clinical improvement. Treatment complications were recorded. It is not clear if this outcome considered patients' symptoms or changes in lesions' appearance or both. It is also unclear how the individual studies were weighted in the meta-analyses. Not all the compared interventions are specified in the meta-analysis for clobetasol.

The authors reported OR of improvement of OLP lesions were greater for patients using topical tacrolimus and clobetasol (OR 8.09 CI 3.77, 17.38; OR 1.21 CI 0.48, 3.05). The authors concluded that topical tacrolimus is more efficacious than clobetasol and may be considered a first-line therapy for management of OLP. Their recommendation lacks a specific concentration and delivery form.

The findings are in disagreement with another systematic review on the topic that used similar criteria and did not find such a magnitude of benefit by using tacrolimus ${ }^{1}$ over clobetasol.

The most commonly reported side effect for clobetasol was candida infections, while local irritation was for tacrolimus. It is not clear what percentage of patients experienced these effects.

Oral lichen planus is a chronic inflammatory disease that affects $1-2 \%$ of the population. It is a T-cell mediated autoimmune process of unknown etiology. Because of its chronic nature and the presence of pain, it is important to provide adequate relief to the patients while minimising complications. In the future it will be important to evaluate patient preferences and have studies with longer follow-ups. Currently tacrolimus is $\mathbf{5 0}$ times more expensive than clobetasol, therefore, cost of treatment should be taken into consideration.

Carol Ann Shipley a and Silvia Spivakovskyb

${ }^{a}$ Monroe County Dental Clinic, Madisonville, Tennessee, USA

${ }^{b}$ New York University College of Dentistry, New York, USA

1 Cheng S, Kirtschig G, Cooper S, Thornhill M, Leonardi-Bee J, Murphy R. Interventions for erosive lichen planus affecting mucosal sites. Cochrane Database Syst Rev 2012; 2: CD008092.

Evidence-Based Dentistry (2016) 17, 16. doi:10.1038/sj.ebd.6401150 